\title{
Internet publicity of data problems in the bioscience literature correlates with enhanced corrective action
}

Several online forums exist to facilitate open and/or anonymous discussion of the peerreviewed scientific literature. Data integrity is a common discussion topic, and it is widely assumed that publicity surrounding such matters will accelerate correction of the scientific record. This study aimed to test this assumption by examining a collection of 497 papers for which data integrity had been questioned either in public or in private. As such, the papers were divided into two sub-sets: a public set of 274 papers discussed online, and the remainder a private set of 223 papers not publicized. The sources of alleged data problems, as well as criteria for defining problem data, and communication of problems to journals and appropriate institutions, were similar between the sets. The number of lab groups represented in each set was also similar (75 in public, 62 in private), as was the number of problem papers per lab (3.65 in public, 3.54 in private). Over a study period of 18 months, public papers were retracted 6.5-fold more, and corrected 7.7 -fold more, than those in the private set. Parsing the results by lab group, 28 labs in the public set had papers which received corrective action, versus 6 labs in the private set. For those labs in the public set with corrected/retracted papers, the fraction of their papers acted on was $62 \%$ of those initially flagged, whereas in the private set this fraction was $27 \%$. Such clustering of actions suggests a pattern in which correction/retraction of one paper from a group correlates with more corrections/retractions from the same group, with this pattern being stronger in the public set. It is therefore concluded that online discussion enhances levels of corrective action in the scientific literature. Nevertheless, anecdotal discussion reveals substantial room for improvement in handling of such matters. 
1 Internet publicity of data problems in the bioscience literature correlates with

2 enhanced corrective action.

3

4 Paul S. Brookes ${ }^{1^{*}}$

5

6 *Address correspondence to: Paul S. Brookes, PhD

Anesthesiology Box 604

8

University of Rochester Medical Center

9

601 Elmwood Avenue,

10

Rochester, NY 14642, USA

11

Tel. 585-273-1626

12

paul_brookes@urmc.rochester.edu

13

$1 \quad$ The author wishes to emphasize that the data collection for this research was conducted outside the boundaries of his position as a University of Rochester faculty member. The author assumes full responsibility for this work, and his affiliation with the University of Rochester does not represent an endorsement of this work by the institution. 
INTRODUCTION

It is widely believed (with somewhat little proof) that the scientific process is inherently self-correcting, and the integrity of the scientific record has always been very important. However, recently the issue of data integrity in published scientific literature has received an unprecedented level of attention both in traditional media outlets such as journal editorial pages [1,2], as well as new media such as blogs and social media websites. A new sub-field has emerged to study the processes that underlie corrections to the scientific literature, and to highlight patterns in the mechanisms of corrective action. retractions in recent years [3], as well as conflicting reports on whether scientific misconduct is a predominant reason for retractions $[4,5]$. With regard to the latter, there is some indication that retraction notices may not be a reliable source of information on underlying causes [4, 6]. Furthermore, although there appears to be a positive correlation between journal impact factor and willingness to retract manuscripts [7], a recent trend toward "mega-corrections" in high impact-factor journals has also been noted [8], suggesting that methods for dealing with problematic data are still evolving.

\section{Blog Roll}

http://abnormalscienceblog.wordpress.com Run by former German scientist Joerg Zwirner. Closed in late 2012 due to personal issues.

http://blog.goo.ne.jp/11jigen Anonymous Japanese blogger Juuichi Jigen, who runs numerous websites, each alleging misconduct by individual scientists.

http://copy-shake-paste.blogspot.com Run by German scientist Debora Weber-Wulff, dealing mostly with plagiarism in academic documents authored by European politicians.

http://retractionwatch.wordpress.com Run by two US science journalists. Covers retractions and surrounding issues. Anonymous comments often include misconduct allegations.

http://www.science-fraud.org Run anonymously by this article's author, reporting on alleged data problems. Site closed by legal threats in Jan 2013.

http://www.pubpeer.com Run by anonymous junior scientists. Permits users to anonymously comment on any paper in the PubMed database.

http://www.ncbi.nlm.nih.gov/pubmedcommons/ NCBI's comment system, currently in beta testing, requires commenters to use a real identity.

As a part of the rapidly developing media landscape in this area, several web-

54 sites have emerged (see "Blog Roll" inset), for readers to post and discuss problematic

55 images and other data, often anonymously. However, this has been met with some 
resistance from established science media outlets [1,9], and several of these sites have been subjected to legal threats $[10,11]$, their proprietors accused of vigilantism [1], and in some cases shut down altogether [10].

With the escalating adoption of social media techniques by science activists, it is critical to ask whether such public discussion of data integrity actually has any effect? Although it is widely assumed that such efforts may enhance the motivation of journals, authors or institutions to take corrective action, this hypothesis has not been rigorously tested. Herein, analysis of nearly 500 papers with alleged data problems reveals a significant corrective effect of enhanced publicity.

\section{METHODS} proprietor of a blog site (see side bar). During this time emails were received to the site, alleging data integrity problems in published journal articles, mostly in the life sciences. These emails were received from individuals using anonymous email accounts, to an anonymous email account protected by two-factor authentication (G-mail), to protect the identity of communicants. Some 274 published papers were documented in blog posts, with specific illustration of the alleged problems, plus relevant background information (e.g., whether authors had other papers retracted/corrected).

In January 2013 legal threats forced the prompt closure of the site, but anonymous submissions continued, and a large quantity of prepared material was left unpublished. This activity yielded a further 223 papers with documented problems, received between November 2012 and January 2013. These papers remained in a private collection.

The 497 papers (274 public + 223 private) all met the same basic criteria for definition of problem data (i.e., documented allegations by an anonymous correspondent, and confirmation of such by myself, using forensic tools such as droplet 83 plug-ins for Adobe Photoshop ${ }^{\mathrm{TM}}$ provided by the US Office of Research Integrity [12]). 84 Anonymous correspondents had also CC'ed journals, authors' host institutions and 85 funding agencies. As such, there was no selection bias present between the public and 86 private papers: all would have eventually been publicized if circumstances had 87 developed differently. 
For each paper, the following parameters were collated into a database: (i)

89 PubMed ID, (ii) Journal, (iii) Year, (iv) Volume, (v) Page \#, (vi) List of problematic data panels, (vii) 5 year impact factor of the journal (2008-2012, ISI Journal Citation Reports), (viii) Outcome. Outcomes were classified into three groups: retraction, publication of an erratum or corrigendum, and no action so far (December 2013). In the case of errata/corrigenda, only those dealing directly with the subject of the questioned data were counted. In addition, only retractions occurring within the time-frame of this study (July 2012 to December 2013) were counted, although it should be noted that some journals do not give reasons for retraction, so attribution of a retraction to a precise cause was not always possible. Supplemental Table 1 shows a list of publications subject to corrective action during the course of this study.

The majority ( $75 \%)$ of problems encountered were apparent inconsistencies in western blotting data (undisclosed splicing, or apparent re-use of bands or blots to represent different experimental conditions), with the remainder relating to apparent reuse of light/fluorescent/electron microscopy images, apparent re-use of text, and apparent re-use of FACS histograms to represent different experimental conditions. Most cases involved data within a single paper, but in a small percentage of cases data appeared to be re-used between papers originating from the same lab' group.

Due to the sensitive nature of its content, the full data set for this study comprising the list of publications, including those for which no action was taken, cannot be provided. A redacted version of the latter was provided for verification purposes during peer review of this study. Where appropriate, statistical differences between groups were determined using ANOVA, and data are presented as means \pm standard deviations with 95\% confidence intervals.

\section{RESULTS}

114 Properties of the public and private paper sub-sets are shown in Table 1. Overall

115 the sets exhibited no differences in number of problematic data panels per paper, or in

116 the 5 year impact factor of the journal they were published in. There was a trend toward

117 papers in the PV group being slightly older, although the reasons for this are not fully 118 understood.

119 For primary outcomes, the public set exhibited a 6.5-fold fold higher rate of 120 retractions, and an 7.7-fold higher rate of corrections, versus the private set. Combined, 
$12123 \%$ of the publicly discussed papers were subjected to some type of corrective action,

122 versus $3.1 \%$ of the private non-discussed papers. This overall 7 -fold difference in levels

123 of corrective action suggests a large impact of online public discussion. Supplementary

124 Table 1 shows the complete list of papers subjected to corrective action.

125 The number of laboratory groups represented was similar between the public and

126 private sets (75 and 62 respectively), as was the average number of identified 127 problematic papers per laboratory group (3.65 public versus 3.54 private). However, 128 despite these similarities, 28 lab' groups in the public set had at least one paper with 129 corrective action taken, versus only 6 lab' groups in the private set. Furthermore, 130 corrective actions appeared to be more clustered in the public set. For lab' groups in this 131 set with corrected/retracted papers, such actions extended to cover almost $2 / 3$ of those 132 initially flagged as problematic (62\%). In contrast, for lab' groups in the private set with 133 corrected/retracted papers, such actions covered little over $1 / 4$ of those initially flagged 134 as problematic (27\%). This suggests that corrective actions in the private set took place 135 on a more individualized basis, with more clustering of corrective actions in the public 136 set perhaps being a direct consequence of greater publicity.

137 Within the public set alone, parsing the papers into outcome groups (Table 2) 138 indicated a trend toward more problematic data panels per paper and lower journal 139 impact factor in the retracted group. In addition a trend toward more recent publication 140 year was seen in both retracted and corrected papers, relative to those for which no 141 action was taken. However, the small sample size (particularly in the retracted paper 142 group) did not permit strong conclusions to be drawn regarding these trends.

\section{DISCUSSION}

145 The primary finding of this study is that online discussion of problematic data is 146 correlated with a nearly 8-fold greater likelihood of either correction or retraction of a 147 paper. This is the first study of its type, and the result should serve as an impetus to 148 encourage further engagement of new media, to push for greater integrity in the 149 scientific literature. In addition, the result suggests that institutions charged with 150 addressing such problems do pay attention to online publicity.

151 In addition, an association was observed between publicity and clustering of 152 corrective actions. Similar numbers of lab' groups were represented in each set, and the 153 number of papers per lab' group initially flagged as problematic was also no different. 
154 Together, these indices suggest that opportunities for corrective action to take place in a 155 clustered manner (i.e. acting on several papers at once) were the same between the 156 public and private paper sets. Nevertheless, more clustering (defined as percentage of 157 total papers flagged for a given lab' group eventually being acted on) was observed in 158 the public set, while corrective actions in the private set appeared to take place more on 159 an individual paper basis. It is possible that publicity was a factor driving this difference 160 i.e., institutions may be more willing to take action on papers if they are aware of other 161 problem papers by the same lab' group, via public discussion forums such as those 162 mentioned earlier. In contrast, if problems identified in papers remain in the private 163 domain, communicated only on an individual basis, then institutions may not see the big 164 picture, and be less willing to take action.

165 The average time from publication to retraction in this study was 4 years, which 166 agrees with previous estimates [13]. However, the observed trend toward greater 167 corrective action for more recently published papers is somewhat counter-intuitive, since 168 it might be expected that newer papers have been read and scrutinized less. This trend 169 could be due to evolving literature consumption patterns among scientists, such that 170 newer papers are more readily available and so read and scrutinized more. Alternatively 171 it may reflect the US Office of Research Integrity's 6 year statute-of-limitations on 172 investigating allegations of misconduct, such that there is less pressure to correct older 173 papers, or insufficient evidence in the form of backup data to prove/disprove any 174 allegations. Finally, this trend toward more corrections in the recent literature could be 175 due to a reported recent uptick in the levels of research misconduct $[3,13]$.

176 Regardless the age of corrected papers, it should be emphasized that the overall 177 levels of corrective action observed in this study are still rather low, at $23.0 \%$ in the 178 public group and only $14.1 \%$ for the complete set of 497 papers. One reason for this 179 (and an important caveat of this study) could be the short study duration of 18 months, 180 such that insufficient time has passed for thorough investigations by journals and 181 institutions. Thus, it will be interesting to revisit these data in future, to see if more 182 papers are corrected. The possibility cannot be ruled out that, given sufficient time, 183 papers in the private set will catch-up to those in the pubic set, although this appears 184 unlikely given current margin between these sub-sets.

185 Another reason for low overall levels of corrective action could be that the alleged 186 problems in these papers are ill-founded and do not warrant action. It is almost 
187 impossible to gauge the magnitude of this problem because the current system of 188 reporting on data integrity only publicizes actionable findings. Journals and institutions 189 often conduct investigations in private and do not disseminate results if no wrongdoing is 190 found. As such, there could be a large number of papers for which a no-fault outcome 191 has been assigned, but this will never be known publicly. Furthermore, counteracting 192 such under-reporting of ill-founded allegations, there are also likely to be cases in which allegations are sound, but retraction or correction notices are insufficiently detailed to 194 indicate this. Thus, even in the case of sound allegations it can often be difficult to provide a solid link between a specific problem in a paper and a course of action taken on it by the journal.

\section{Anecdotal evidence of a corrective system in need of improvement...}

Case 1: I contacted a journal anonymously to highlight problems in 5 papers. 15 subsequent emails to the journal, several editorial board members, and the governors of the scientific society that oversees the journal, all failed to elicit a single response, even to acknowledge correspondence. Refusal to communicate is contrary to guidelines of the Committee on Publication Ethics (COPE) which the journal is a member of.

Case 2: I reviewed a paper and found fabricated data. The journal rejected the paper, and subsequently it was published in a different journal with some problem data still present. The editor at the new journal knows about the previous rejection for reasons of data fabrication, but refuses to take up the matter with the authors unless I am willing to have my real name revealed as an accuser. I refused, because the lead author is on a panel that reviews my grant proposals.

Case 3: Two multi-panel figures were duplicated in their entirety, including figure legends and descriptive text, in two papers in different journals, submitted a week apart. Both journals permitted authors (who have retracted 2 other papers for acknowledged misconduct) to issue a correction, merely stating the data were the same. COPE guidelines, to which both journals subscribe, are quite clear regarding dual submission of data to more than one journal.

Case 4: I reported on fabricated data in a supplementary file. 3 months later a blog commenter (whose IP address resolved to the city of the lead author) claimed the report was incorrect, and demanded its removal. Coincidentally, that same day the journal website had posted a new supplemental data file, with the problem data replaced, but no correction notice. I contacted the journal, but more than a year later they have not acknowledged the correction took place.

Another reason for low levels of corrective action is suggested by anecdotes (see

218 inset) indicating that journals and other institutions may not wish to engage in dealing 219 with such matters. Many journals do not respond to allegations from anonymous 220 correspondents as a matter of policy, and while there are several reasons for this (e.g., 221 not wishing to allow scientific competitors to sabotage rivals' work), it is clear that 222 journals do have some lee-way in determining whether to respond to anonymous 223 correspondents. Aside from the issue of anonymity, these anecdotes and the notes on 224 Supplemental Table 1 are diagnostic of a corrective system that is far from perfect. While 225 it is beyond the scope of this manuscript to speculate on ways to improve the corrective 226 system in the scientific literature, recent developments such as PubPeer and PubMed 
227 Commons are seen as steps in the right direction, toward universal and open post228 publication peer review.

With discussions ongoing in the scientific community regarding post-publication peer review, there appears to be little agreement overall on the issue of anonymity. 231 While anonymity is often beneficial for junior scientists (who may for example fear 232 repercussions when raising questions about a senior scientists' work), a purely 233 anonymous system is also open to abuse (e.g. sabotage of colleague's work). A 234 moderated discussion system may help to avoid such abuses, although in the current 235 fiscal climate it is unlikely that sufficient funds exist to pay for moderators, who would necessarily have to be highly trained in scientific sub-fields. somewhat small sample size, particularly for the retracted group of papers. (ii) The data for the study came from a limited number of anonymous correspondents and concerned mostly problems with image manipulation in life-sciences papers. As such, it is not clear if the patterns observed herein are generalizable to the scientific literature at large. (iii) The study was not prospectively designed, and although every care was taken to conduct it in an ethically sound and unbiased manner, the research was conducted by the author as a private citizen and therefore fell outside of university institutional review board (IRB) oversight. While it is not immediately obvious that such research would even fall under the topic of human subjects research, the anonymity of correspondents reporting on papers was strictly maintained, and to date all remain anonymous to the author. (iv) Every effort was made to ensure that problems identified were communicated adequately to the appropriate parties, but this could not be verified for every single paper. In some cases, the only evidence supporting knowledge of a problem by a journal or institution, was the word of an anonymous email correspondent. Attempts to verify such information were rendered difficult by non-disclosure policies surrounding ongoing investigations, and this information is likely impossible to verify completely. (v) While the author has made efforts to make the data set available to the

255 fullest extent possible during peer review, clearly these data are of a sensitive nature, 256 and as such it is unlikely that the study can be reproduced independently. (vi) There are 257 likely to be unknown and uncorrected factors that were different between the 258 public/private paper sets. These could include subtle differences in scientific sub-field 259 between the sets (e.g., cancer vs. neurology) or the precise make-up of sub-fields or 
260 nationalities to which the anonymous correspondents belonged in each set. While it is

261 unlikely such factors will ever be fully resolved, the large difference in primary outcomes

262 between papers discussed online and those not (i.e., nearly 8-fold greater levels of 263 corrective action), suggests this result is unlikely due to such factors entirely.

264 In summary, the current study shows that publicity surrounding issues of 265 problematic data has a large impact on subsequent actions to correct the scientific 266 record. Nevertheless, anecdotal evidence suggests there is substantial room for 267 improvement in the standards for dealing with such issues at the institutional and 268 publisher levels.

\section{ACKNOWLEDGEMENTS}

The author is grateful to R. Grant Steen (MedicC!, Medical Communications 272 Consultants), Ferric Fang (University of Washington), Laurent Glance, and Igor 273 Milosevic (University of Rochester Medical Center), and members of his laboratory for 274 stimulating discussions during the preparation of this manuscript. In the author thanks 275 both anonymous and named reviewers and editors at Peer $\mathrm{J}$, for suggestions that 276 greatly improved the manuscript. 


\section{LITERATURE CITED}

278 [1] Mole VIII. The end of science. J Cell Sci 2012, 125: 5907-5909.

279 [2] Bosch X. Research integrity: Journals should be clear on misconduct. Nature 2013, 280 497: 40.

281 [3] Steen RG, Casadevall A, Fang FC. Why Has the Number of Scientific Retractions 282 Increased? PLOS ONE 2013, 8: e68397.

283 [4] Fang FC, Steen RG, Casadevall A. Misconduct accounts for the majority of retracted 284 scientific publications. Proc Natl Acad Sci USA 2012, 109: 17028-17033.

285 [5] Grieneisen ML, Zhang M. A Comprehensive Survey of Retracted Articles from the 286 Scholarly Literature. PLOS ONE 2012, 7: e44118.

287 [6] Resnik, DB, Dinse GE. Scientific retractions and corrections related to misconduct 288 findings. J Med Ethics 2013, 39: 46-50.

289 [7] Fang FC, Casadevall A. Retracted Science and the Retraction Index. Infect Immun 290 2011, 79: 3855-3859.

291 [8] Oransky I, Marcus A. We're Mostly Wrong but Trust Us. Is the "mega-correction" 292 here to stay? Lab Times 2012, 2012_01: 43.

293 [9] Weiss PS et al. (ACS Nano Editorial Board). Be Critifal but Fair. ACS Nano 2013, 7: 294 8313-8316.

295 [10] Couzin-Frankel J. Author of Popular Blog That Charged Fraud Unmasked. Science 296 2013, 339: 132.

297 [11] http://www.popehat.com/2013/04/11/

298 [12] http://ori.hhs.gov/droplets

299 [13] Steen RG Retractions in the scientific literature: is the incidence of research fraud 300 increasing? J Med Ethics 2011, 37: 249-253. 


\section{Table 1 (on next page)}

Table 1

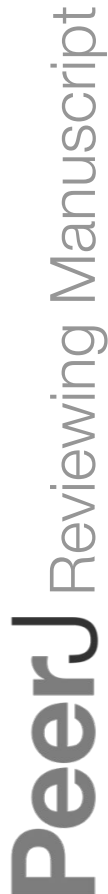


Table 1. Properties of papers in the public and private sub-sets. Means \pm standard deviations, with 95\% confidence intervals where appropriate.

\begin{tabular}{lcc}
\hline & Public & Private \\
\hline \# papers & 274 & 223 \\
\# Retractions, (\%) & $16(5.8)$ & $2(0.9)$ \\
\# Corrections, (\%) & $47(17.2)$ & $5(2.2)$ \\
\# of problematic data panels/paper (95\% & $2.3 \pm 1.7(2.1-2.5)$ & $2.5 \pm 1.5(2.3-2.7)$ \\
Cl) & $9.3 \pm 8.5(8.3-10.3)$ & $8.7 \pm 7.1(7.7-9.6)$ \\
5 yr. Journal Impact Factor (95\% Cl) & $2007.5 \pm 4.3$ & $2004.8 \pm 4.1$ \\
Publication year & 75 & 62 \\
Total \# of lab' groups & $3.65 \pm 3.61(2.79-$ & $3.54 \pm 5.16(2.26-4.82)$ \\
\# of problematic papers / group & $4.42)$ & 6 \\
\# of lab' groups with action on papers & 28 & \\
Papers with action, as \% of those & $62.4 \pm 31.5(50.8-$ & $26.8 \pm 26.1(5.9-47.7)$ \\
$\quad$ flagged for a given lab' group (95\% & $74.1)$ & \\
\hline
\end{tabular}




\section{Table 2 (on next page)}

Table 2

Table 2. Selected properties of papers in the public set, broken down by outcome. Means \pm standard deviations, with 95\% confidence intervals where appropriate. 
Table 2. Selected properties of papers in the public set, broken down by outcome. Means \pm standard deviations, with 95\% confidence intervals where appropriate.

\begin{tabular}{lccc}
\hline & Retracted & Corrected & No Action \\
\hline \# papers ${ }^{\text {a }}$ & 16 & 47 & 212 \\
$\begin{array}{l}\text { Problematic data } \\
\text { panels/paper } \\
\quad(95 \% \text { CI) }\end{array}$ & $3.1 \pm 38$ & $2.2 \pm 1.4$ & $2.2 \pm 1.4$ \\
5 yr. Journal Impact Factor & $(2.5-4.2)$ & $(1.8-2.7)$ & $(2.0-2.4)$ \\
$\quad(95 \%$ Cl) & $6.9 \pm 3.7$ & $10.0 \pm 8.6$ & $9.3 \pm 8.7$ \\
& $(5.2-8.6)$ & $(7.5-12.4)$ & $(8.2-10.5)$ \\
Publication year & $2008.3 \pm$ & $2009.3 \pm$ & $2007.1 \pm$ \\
& 4.4 & 3.0 & 4.4 \\
\hline $\begin{array}{l}{ }^{a} \text { Total }=275, \text { not 274 as expected, since one paper was corrected and subsequently } \\
\text { retracted. }\end{array}$ & & & \\
\hline
\end{tabular}

